\title{
Modeling of Drift Effects on Solar Tower Concentrated Flux Distributions
}

\author{
Luis O. Lara-Cerecedo, ${ }^{1}$ Isaias Moreno-Cruz, ${ }^{2}$ \\ Nun Pitalúa-Diaz, ${ }^{1}$ and Camilo A. Arancibia-Bulnes ${ }^{2}$ \\ ${ }^{1}$ Departamento de Ingeniería Industrial, Universidad de Sonora, Boulevard Encinas y Rosales s/n, Colonia Centro, \\ 83000 Hermosillo, SON, Mexico \\ ${ }^{2}$ Instituto de Energías Renovables, Universidad Nacional Autónoma de México, Privada Xochicalco s/n, 62580 Temixco, MOR, Mexico \\ Correspondence should be addressed to Camilo A. Arancibia-Bulnes; caab@ier.unam.mx
}

Received 4 December 2015; Accepted 23 February 2016

Academic Editor: Manuel Ignacio Maldonado

Copyright ( $) 2016$ Luis O. Lara-Cerecedo et al. This is an open access article distributed under the Creative Commons Attribution License, which permits unrestricted use, distribution, and reproduction in any medium, provided the original work is properly cited.

A novel modeling tool for calculation of central receiver concentrated flux distributions is presented, which takes into account drift effects. This tool is based on a drift model that includes different geometrical error sources in a rigorous manner and on a simple analytic approximation for the individual flux distribution of a heliostat. The model is applied to a group of heliostats of a real field to obtain the resulting flux distribution and its variation along the day. The distributions differ strongly from those obtained assuming the ideal case without drift or a case with a Gaussian tracking error function. The time evolution of peak flux is also calculated to demonstrate the capabilities of the model. The evolution of this parameter also shows strong differences in comparison to the case without drift.

\section{Introduction}

Solar thermal electricity production is carried out by means of sun tracking concentrating mirrors. These are employed to achieve the high temperatures required by thermodynamic processes, like Rankine or Brayton cycles. The most promising is central receiver technology, which is an old idea, but only recently it has been applied commercially [1-4]. Technical challenges remain to realize the full potential of this technology.

Central receiver (CR) plants use a large number of twoaxis tracking heliostats to focus the sun. These mirrors concentrate radiation in the receiver, which is at the top of a tower, up to $140 \mathrm{~m}$ high. Depending on the capacity of the plant several hundreds to tens of thousands of these movable mirrors are used, which can be located at distances of up to $1500 \mathrm{~m}$ from the tower. The efficient operation of CR plants is strongly dependent on the quality of the concentrated solar beam produced jointly by all of those individual mirrors. So, the tracking control is necessary to keep a good aiming of heliostats, in order to achieve desired radiative flux or temperature distributions $[5,6]$.

Heliostat control requires adjusting the angles of the tracking mechanism according to calculations in such a way that the heliostat normal vector bisects the angle between solar vector and relative target position vector [7]. However, this task is subject to many errors [8-16]. Drift is defined as the wandering of the concentrated light spot produced by a heliostat. The different types of drift and their causes have been discussed in detail $[8,17]$. This leads to a statistical distribution of tracking errors that has been shown to differ from the conventional Gaussian behavior [9].

Drift is always present in CR plants. It can be reduced by different time consuming methodologies [18], but never completely eliminated.

To optimize the design of a CR plant, it is important to analyze the optical behavior of the heliostat field, which has created the need for specialized modeling tools. There are different methods that have been proposed to evaluate the flux distribution produced by heliostats [19]. In particular, 


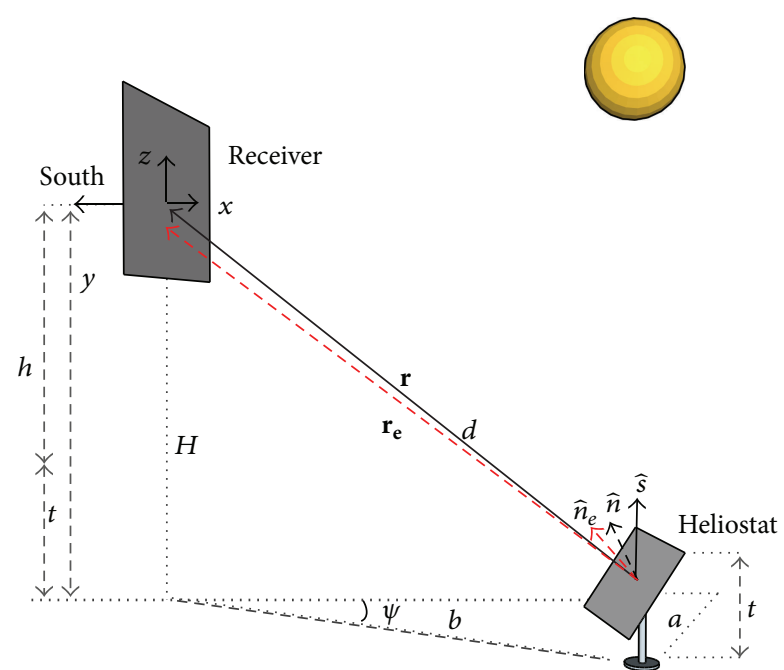

FIGURE 1: Geometry of the problem.

Collado et al. [20] presented an analytical model for the flux density of a heliostat that focuses on a target in a central tower plant. By this model an exact convolution is obtained, considering spherical and continuous surface of the mirror, linear conformal transformation in the complex plane, equivalent to the reflection mapping between an on-axis aligned heliostat and the objective located on the receiver, circular Gaussian distribution of the effective sunshape, and the concentration function constant. This model allows getting an analytic flux density function of the energy reflected by a heliostat.

The analytical model of Collado et al. [20] is able to acceptably reproduce the partial results of discrete evaluations. Therefore, the model could be appropriate for quick preliminary estimations and primary optimizations [21]. Walzel et al. [22] found that the accuracy of codes using convolution techniques, the peak flux error, and average absolute error is in the range of $1-2 \%$, which is comparable to what can be obtained from ray-tracing codes like SolTrace.

All the above simulation methodologies assume that the imperfect tracking of heliostats can be taken into account in simulations by considering long term average behavior based on a Gaussian distribution of tracking errors. The major drawback of this approach is that it is not useful at all to analyze the dynamic effects that may appear in the daily operation of a solar tower power plant. Therefore its usefulness is limited to the design of the plant.

In the present work a rigorous geometrical model of drift behavior $[8,9]$ is coupled to the very fast heliostat flux model described above [20]. This allows developing a method to study the dynamic behavior of the flux distribution, including its uniformity and average distribution.

\section{Methodology}

To analyze the dynamical behavior of flux distribution in the receiver of a solar tower two elements are needed: a drift model, describing the imperfect tracking by the heliostats, and a heliostat flux distribution model to evaluate the individual contribution of each heliostat according to its deviated centroid on the target.

2.1. Drift Simulation. The methodology for drift calculations has been discussed elsewhere [8], so here it will be only briefly outlined.

The ideal normal vector of the heliostat at any time is given by the solar position vector and relative heliostat to target vector (Figure 1) and the law of reflection:

$$
\widehat{n}=\frac{\widehat{r}+\widehat{s}}{\|\widehat{r}+\widehat{s}\|}=(\sin \beta \sin \gamma, \sin \beta \cos \gamma, \cos \beta),
$$

where $\beta$ and $\gamma$ are the elevation and azimuth angles of the heliostat, respectively, and

$$
\widehat{r}=\left(a^{2}+b^{2}+h^{2}\right)^{-1 / 2}(-a, b, h) .
$$

The solar unit vector is given by the zenith and azimuth angles, calculated according to the expressions presented by Duffie and Beckman [23]:

$$
\widehat{s}=\left(\sin \theta_{z} \sin \gamma_{s}, \sin \theta_{z} \cos \gamma_{s}, \cos \theta_{z}\right) .
$$

The different tracking errors modify the ideal normal vector of (1), and as a consequence the reflected vector is also modified. The latter can be calculated by another form of the law of reflection [24]:

$$
\widehat{r}_{e}=-\widehat{s}+2 \widehat{n}_{e}\left(\widehat{n}_{e} \cdot \widehat{s}\right),
$$

where $\widehat{n}_{e}$ is the normal vector, modified by the tracking errors, and $\widehat{r}_{e}$ is unit direction vector of the resulting reflected ray. The impact point of the ray reflected at the center of the heliostat on the receiver is used to describe the drift. This impact point is determined from the intersection of reflected vector with a vertical plane located at the front face of the tower, representing the receiver.

Three drift mechanisms are analyzed here (Figure 2): angular reference offset, heliostat tilt, and canting error.

The angular reference offset error is a constant offset in the drive mechanism. In this case the heliostat elevation and azimuth angles at any given time have a constant shift $\left(\beta^{\prime}, \gamma^{\prime}\right)=\left(\beta+\Delta \beta_{e}, \gamma+\Delta \gamma_{e}\right)$ instead of the ideal values $(\beta, \gamma)$ that can be obtained from the ideal normal vector given equation (1).

Canting error is a deviation of the optical axis of the heliostat from perpendicularity with respect to elevation axis (Figure 2(b)) by an angle. To obtain the expression for the deviated normal vector in this case, consider a global reference system with coordinate axes $(x, y, z)$ and a local reference system, centered in the ideal heliostat plane, with axes $(u, v, w)$, as shown in Figure 3.

The canting error of a heliostat involves a deviation in the normal vector that should in principle be represented by two angles, measured over the planes $(u, w)$ and $(v, w)$. However, the error angle in the plane $(v, w)$ rotates around the elevation axis. Thus, it is actually indistinguishable on its effects from an offset error around this axis, and therefore it 


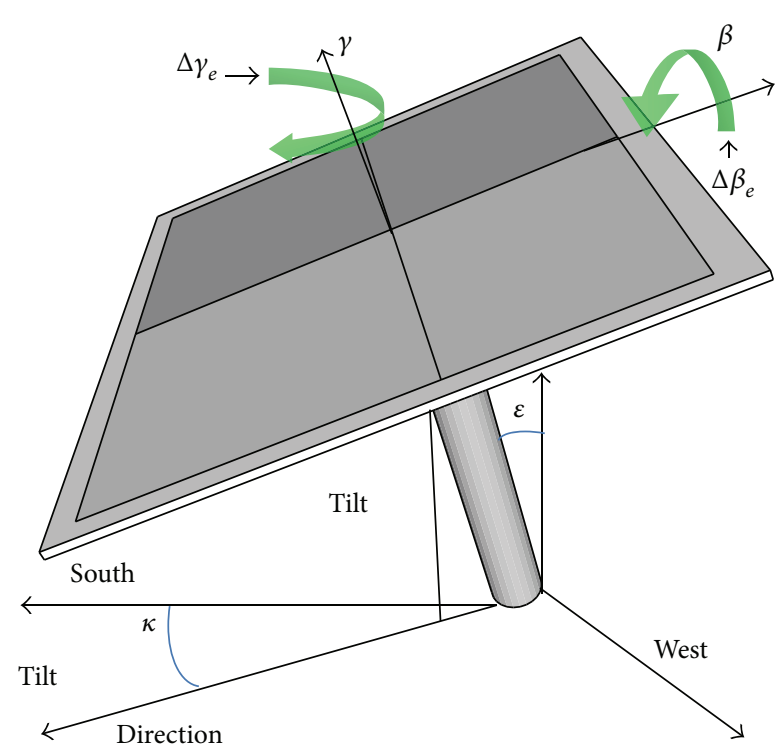

(a)

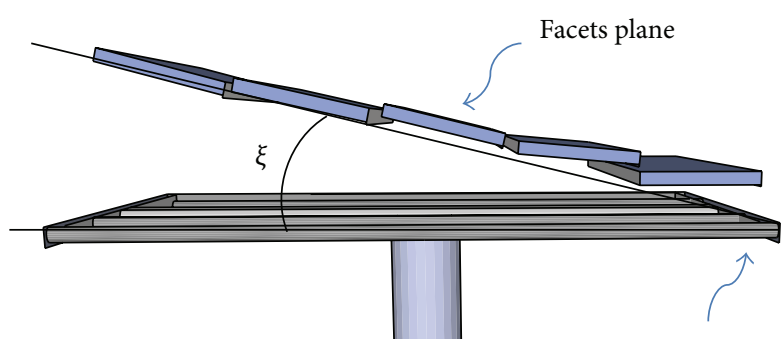

Elevation axis

Figure 2: Primary error sources: angular reference offset and inclination of the azimuth rotation plane due to pedestal or gearbox tilt (a); canting error (b).

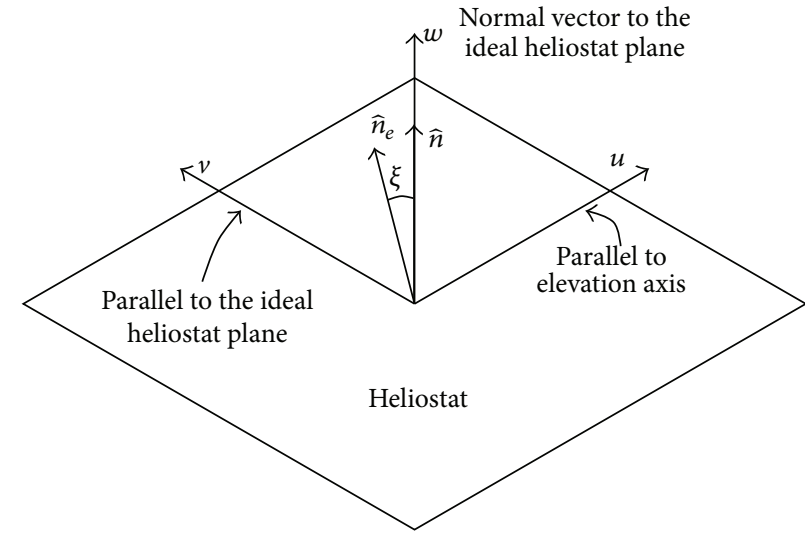

(a)

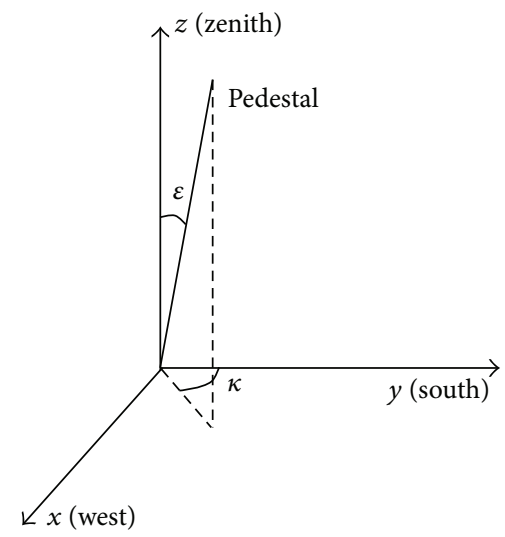

(b)

FIGURE 3: The coordinate system (a) shows the canting error in the local system. The coordinate system (b) shows the global reference.

is included already in the parameter $\Delta \beta_{e}$. The deviation in plane $(u, w)$ is described by the angle $\xi$. Thus, in the local reference system the deviated normal vector can be expressed as $\widehat{n}_{e}=(-\sin \xi, 0, \cos \xi)$. To obtain this vector in the global reference system a rotation should be applied corresponding to the actual angles of the mechanisms of the heliostat, $\beta^{\prime}=$ $\beta+\Delta \beta_{e}$ and $\gamma^{\prime}=\gamma+\Delta \gamma_{e}$. The rotation matrix is given by

$$
P=\left(\begin{array}{ccc}
-\cos \gamma^{\prime} & -\cos \beta^{\prime} \sin \gamma^{\prime} & \sin \beta^{\prime} \sin \gamma^{\prime} \\
\sin \gamma^{\prime} & \cos \beta^{\prime} \cos \gamma^{\prime} & \sin \beta^{\prime} \cos \gamma^{\prime} \\
0 & \sin \beta^{\prime} & \cos \beta^{\prime}
\end{array}\right) .
$$

Then the deviated normal vector, including canting error, in the global reference system is given by

$$
\begin{aligned}
\widehat{n}_{e, \mathrm{oc}} & =\left(\sin \beta^{\prime} \sin \gamma^{\prime} \cos \xi\right. \\
+ & \cos \gamma^{\prime} \sin \xi, \sin \beta^{\prime} \cos \gamma^{\prime} \cos \xi \\
& \left.-\sin \gamma^{\prime} \sin \xi, \cos \beta^{\prime} \cos \xi\right) .
\end{aligned}
$$

The third error, gearbox tilt, is caused by a nonvertical azimuth rotation axis (Figure 2(a)). The tilt angle is $\varepsilon$ and the tilt direction is $\kappa$. Then, the deviated normal vector is 
finally given by a rotation matrix applied to the normal vector, distorted by the previous two errors (see (6)):

$$
\begin{aligned}
& \widehat{n}_{e}=M \widehat{n}_{e, 0 \mathrm{c}}, \\
& M \\
& =\left(\begin{array}{ccc}
\cos \varepsilon \sin ^{2} \kappa+\cos ^{2} \kappa & (\cos \varepsilon-1) \cos \kappa \sin \kappa & \sin \varepsilon \sin \kappa \\
(\cos \varepsilon-1) \cos \kappa \sin \kappa & \cos \varepsilon \cos ^{2} \kappa+\sin ^{2} \kappa & \sin \varepsilon \cos \kappa \\
-\sin \varepsilon \sin \kappa & -\sin \varepsilon \cos \kappa & \cos \varepsilon
\end{array}\right) .
\end{aligned}
$$

This expression is obtained as the result of a series of three rotations by the angles $\kappa$, around a vertical axis, $\varepsilon$, around a horizontal axis, and $-\kappa$, around a vertical axis.

2.2. Flux Distribution. As was discussed in the introduction, different methods exist [19] for the simulation of the flux distribution produced by heliostats. However in this work we utilize the method proposed by Collado et al. [20], due to its clear advantage in computational speed.

The used method is based on assuming continuous heliostat facets of spherical curvature, reflecting Gaussian beams towards the heliostat, and assuming a Gaussian sun. The flux distribution produced by a heliostat can be expressed as

$$
F(x, z)=C I \rho H\left(\zeta,-a_{1}, a_{1}\right) H\left(\eta,-a_{2}, a_{2}\right) .
$$

In the above expression, the function $H$ is defined in terms of two error functions

$$
H(w, a, c)=\left(\frac{1}{2}\right)[\operatorname{erf}(w-a)+\operatorname{erf}(w-c)] .
$$

This kind of expression is reached after integrating the Gaussian contributions on the surface of the heliostat and considering rotations and translations between the local reference system of the heliostat and the global reference system. So the above generalized coordinates $(\zeta, \eta)$ are related to coordinates on the screen $(x, z)$ by

$$
\begin{aligned}
\zeta & =\frac{x^{\prime}}{\left(\sqrt{2} \sigma_{r}\right)}, \\
\eta & =\frac{z^{\prime}}{\left(\sqrt{2} \sigma_{r}\right)}, \\
x^{\prime} & =x \cos (\phi-\tau)+z \sin (\phi-\tau), \\
z^{\prime} & =z \cos (\phi-\tau)-x \sin (\phi-\tau) .
\end{aligned}
$$

The parameters $a_{1}$ and $a_{2}$ are related to the width $L_{w}$ and height $L_{h}$ of the heliostat

$$
\begin{aligned}
& a_{1}=\frac{L_{w}}{\left(\sqrt{2} \sigma_{r}\right)}, \\
& a_{2}=\frac{L_{h}}{\left(\sqrt{2} \sigma_{r}\right)} .
\end{aligned}
$$

The meaning of the rest of the parameters and rotation angles $(\phi$ and $\tau$ ) appearing in the above expressions can be consulted in [20], since their development is too cumbersome to be discussed here.

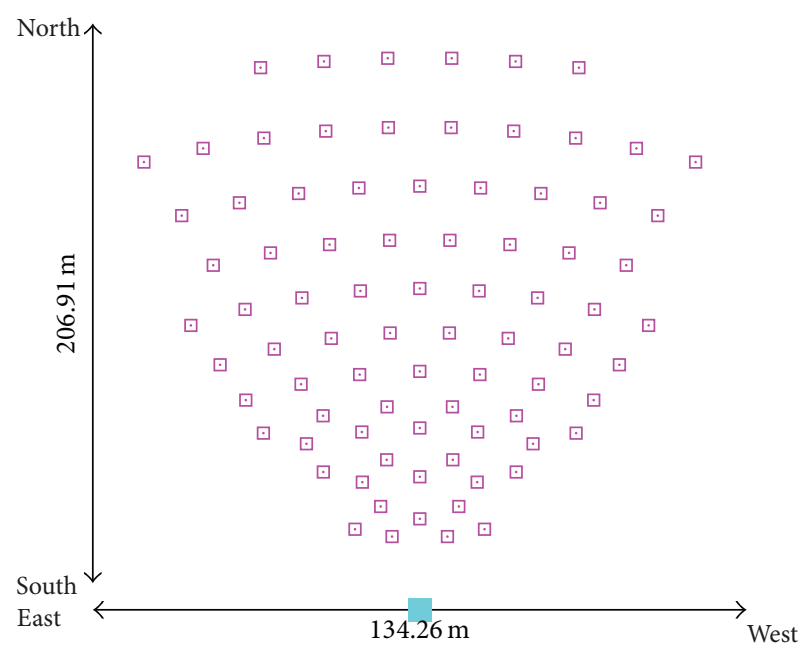

FIgure 4: Layout of the heliostat field. The receiver, located at the north face of the tower, is centered at $(0,0,32)$.

2.3. Coupling of the Methods. The coupling of the drift model and the flux distribution model is carried out through the deviated normal vector $\widehat{n}_{e}$ and the centroid impact point. These are directly introduced into Collado's et al. mathematical method for flux calculation. First a drift simulation is carried out for a whole day for some heliostats in the field. Then, the next step is to obtain the normal vectors with errors and impact points from the heliostats simulated. These will be transferred to the routine for flux simulation.

Once these drift errors have been applied, each individual heliostat flux distribution is simulated for every time step, and then they are added to obtain the resulting flux distribution on the receiver.

\section{Results and Discussion}

In this section results are presented for the analysis of the impact of the proposed drift mechanisms on the flux distributions. The analyzed variables are the flux distributions themselves, their uniformity and centroid. All simulations are carried out for latitude of $29^{\circ}$ north, corresponding approximately to the location of Hermosillo, Mexico. The heliostat field will be the Central Receiver Experimental Field of UNISON and UNAM, located in Hermosillo. This field is illustrated in Figure 4.

First of all, it is interesting to compare the results of the analytical methodology proposed by Collado et al. [20] with more rigorous Monte Carlo results obtained with the SolTrace software. In Figures 5 and 6 two particular cases are shown comparing the obtained flux distributions of individual heliostats in the field. In Table 1, a systematic comparison of peak and average flux for both methods is shown for 10 different heliostats located in different parts of the field.

As can be observed in the examples presented in Figures 5 and 6 and Table 1, there is a very good coincidence between the rigorous (but noisy) results obtained with the MC method and the approximate results of the implemented analytical method. Maximum differences in the peak flux can be up 


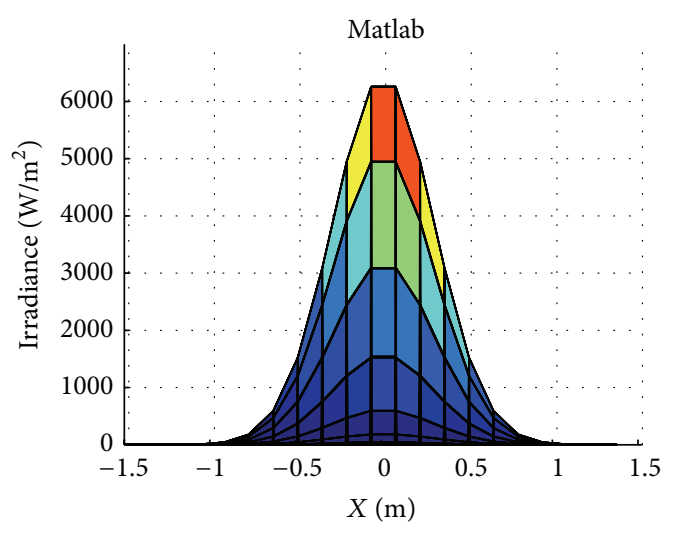

(a)

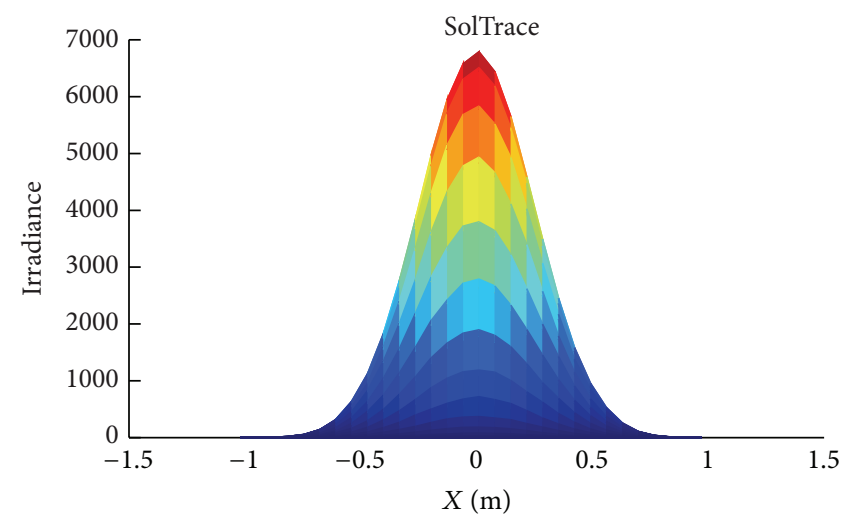

(b)

FIGURE 5: Comparative of solar flux in the receiver. Analytical approximation (a) and Monte Carlo method using SolTrace (b). Close heliostat located at $(0,34.13)$.

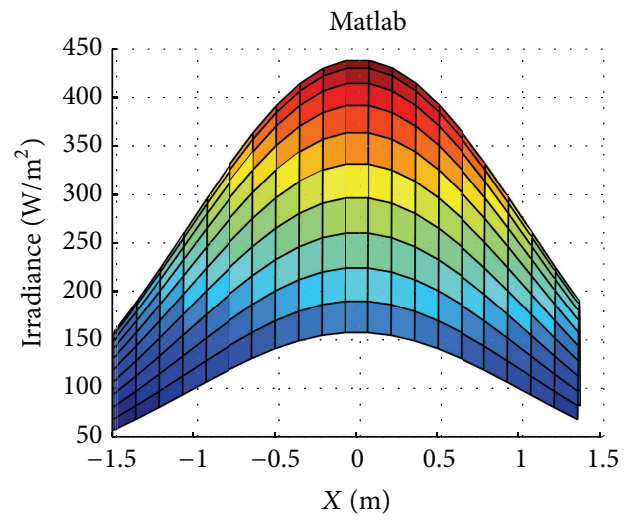

(a)

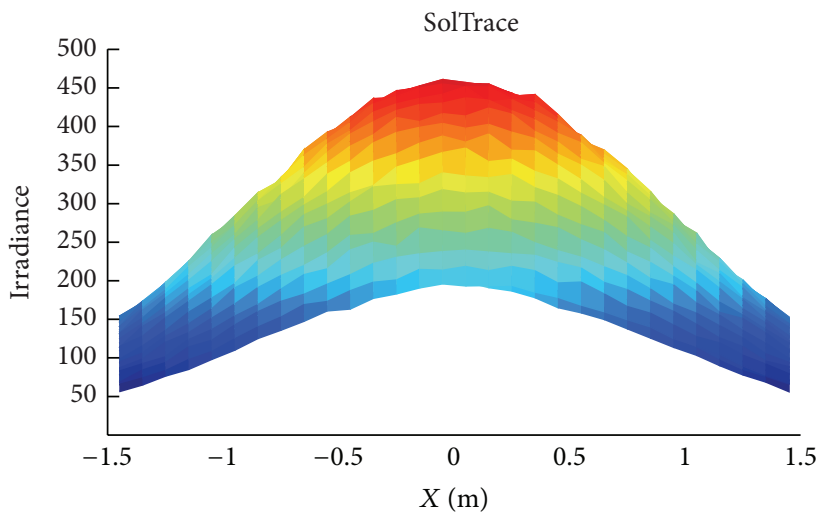

(b)

FIgure 6: Comparative of solar flux in the receiver. Analytical approximation (a) and Monte Carlo method using SolTrace (b). Far heliostat located at $(-67.5,167.99)$.

to $5 \%$, while in the average flux they are around $2.5 \%$. It is very interesting to observe that the differences seem to increase with distance, contrary to what one may expect from the nature of the model, which ignores somewhat the actual geometry of the heliostat.

Moreover the coincidence of the shape of the curves is remarkable, which suits very well the need for a fast calculation tool that allows studying the joint flux distribution of the drifting heliostats.

In Figure 7 the drift behavior is presented for a complete day simulation on summer solstice for four representative error sets. This is for 4 heliostats in different positions of the field. As explained before, each of these trajectories represents the daily wandering of the centroid of the solar spot produced by a heliostat. It can be observed that a variety of different trajectories can be observed depending on the values of the input error parameters and have been discussed in more detail elsewhere [8].

The next step, as discussed, is the coupling of the drift simulation with the flux distribution. A result for a collection of 5 heliostats is presented in Figure 8. For simplicity to avoid excessive differences in spot size for the different heliostats, all of them have been considered to be at the same distance of $120 \mathrm{~m}$, but spanning a range of angles with respect of the north-south axis of the field (angle $\psi$ ).

The cases presented in Figure 8 correspond to a case with drift. We can see that at 7:00 all heliostat images coincide exactly, but as the day advances drift is observed. At 8:00 the flux distribution is starting to deform, and at 10:00 two heliostats are clearly separating from the group. One of them is rapidly isolated and does not contribute to the peak flux anymore. However, at least three heliostats are partially overlapping for the whole day. By 15:00, the peak flux has decreased significantly, due to the spreading.

The effect of the above behavior on the peak flux for the whole day is presented in Figure 9, for both the ideal and the drift cases. Huge differences can be appreciated, where the peak flux never reaches 20 suns for the case with drift. For the ideal case still a variation in peak flux can be observed, which is rather a result of DNI variation and the cosine effect, due to the larger angles subtended by the sun and the target at the beginning and end of the day. 

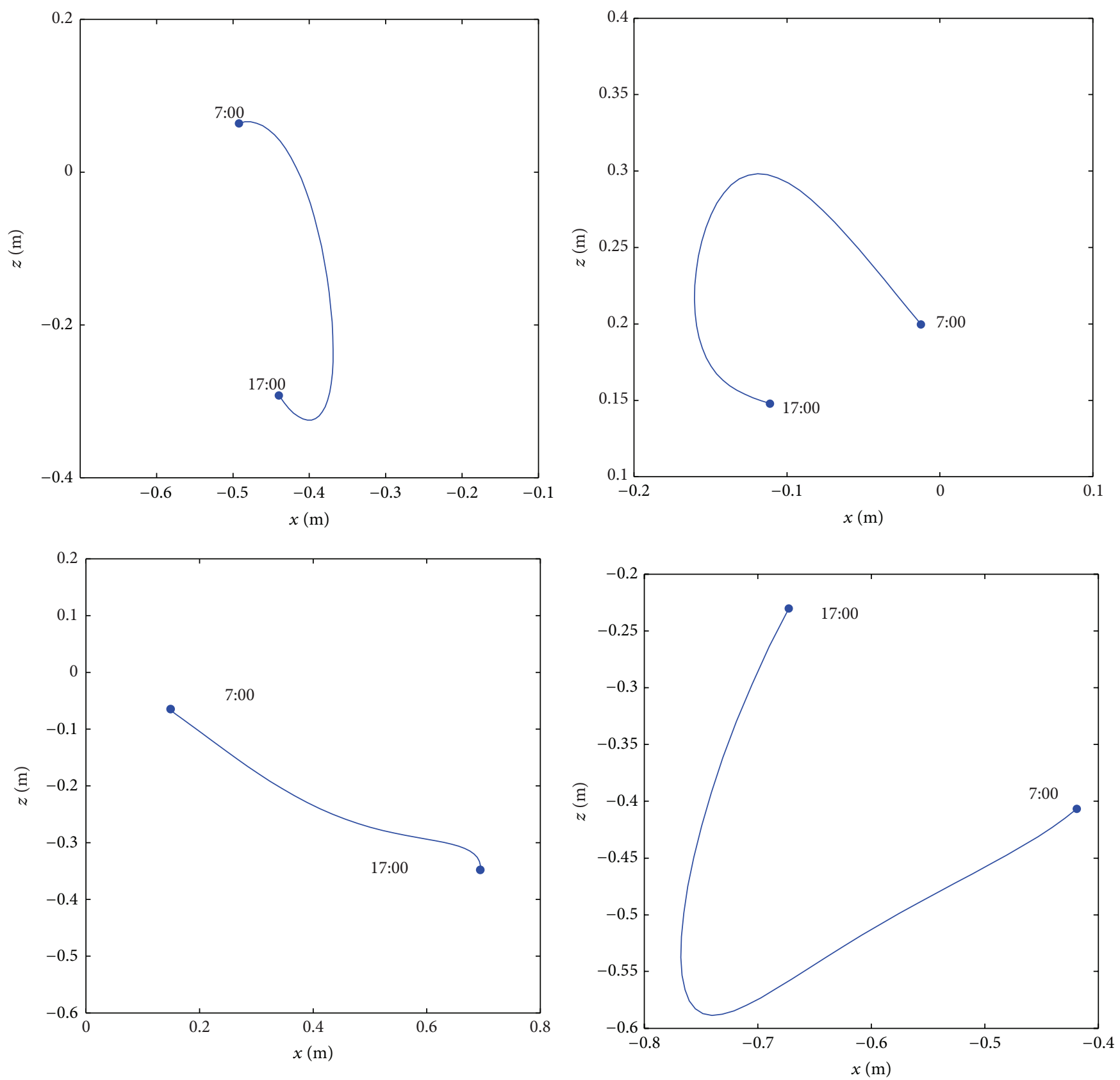

FIGURE 7: Comparative of image centroid drift curves for individual heliostats. Summer solstice. All errors are between -3 and 3 mrad.

It is important to point out that drift in general takes the images out of the center of target, as seen in Figure 7. In fact, it is common to apply a constant compensation [18], which centers the trajectories on the target but does not correct drift. More sophisticated correction methods require much more implementation effort [20]. In Figure 8 a constant compensation control strategy is applied. Thus the spots coincide at the beginning of the day, but their coincidence worsens as the day advances.

Further discussion about aim and limitations of the proposed model becomes necessary at this point. First of all, let us point out that the objective of this tool is not to be a predictive model by any means or capable of actually producing simulations directly comparable with measured flux distributions. The task of evaluating the set of primary error parameters $\left(\xi, \varepsilon, \kappa, \Delta \beta_{e}, \Delta \gamma_{e}\right)$ for all the heliostats in real solar field is not practical or economically feasible. Thus it is very hard to obtain the information required to actually simulate the combined drift behavior of many heliostats in a real field. On the other hand, the Collado et al. approximation, based on the very idealized profiles given by (8), is not a realistic representation of the irradiance distribution of 

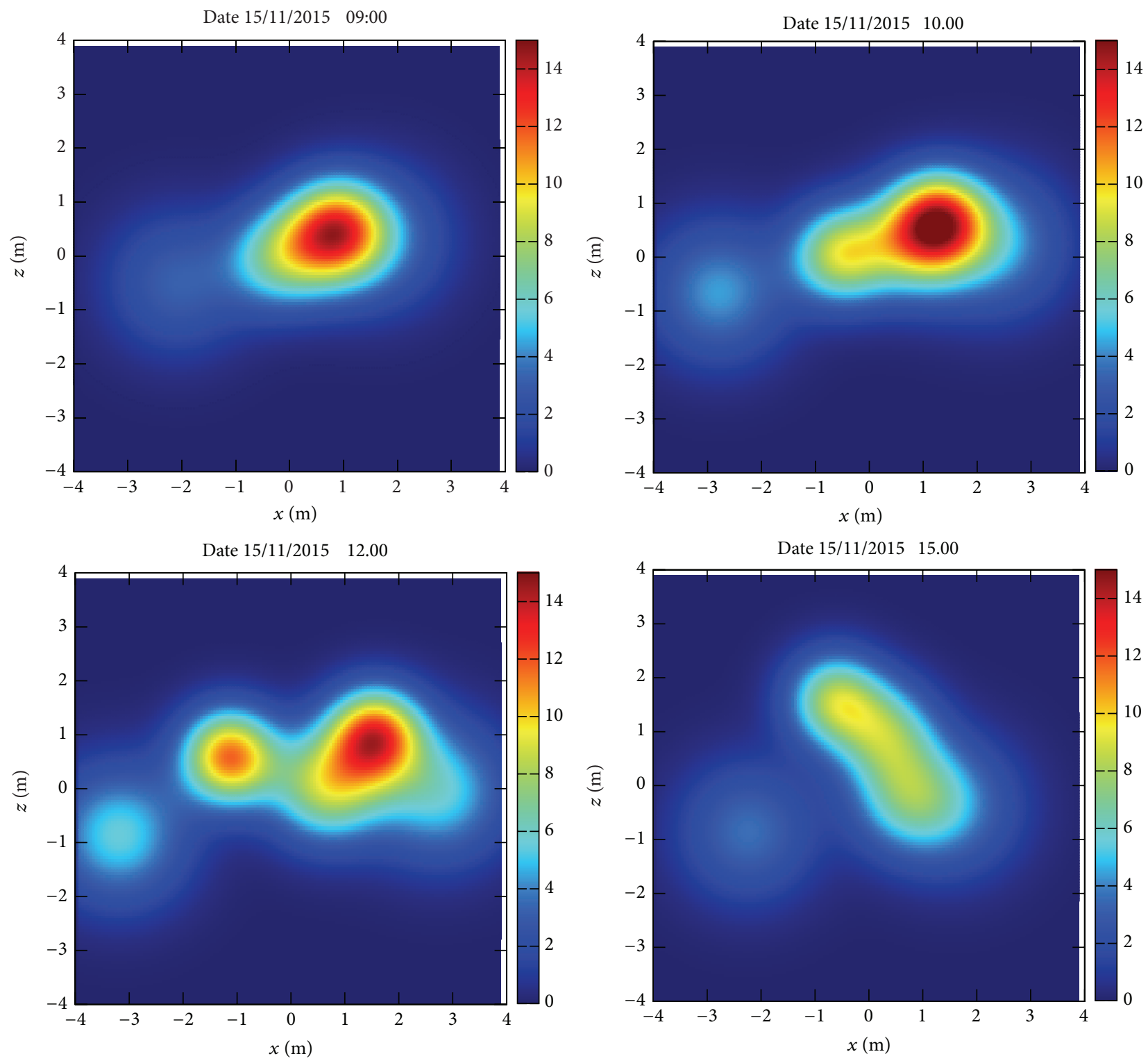

FIGURE 8: Flux distributions on the receiver for 5 heliostats in a case with drift, for the indicated hours and date, at local time of Hermosillo, Sonora, Mexico. The color scale expresses irradiance in units of $\mathrm{W} / \mathrm{m}^{2}$.

TABLE 1: Comparative of peak fluxes and average fluxes of analytical method using Matlab and Monte Carlo method by using SolTrace.

\begin{tabular}{|c|c|c|c|c|c|c|}
\hline $\begin{array}{l}\text { Heliostat } x, y \\
\text { coordinates } \\
(\mathrm{m})\end{array}$ & $\begin{array}{c}\text { Peak flux } \\
\mathrm{MC}\left(\mathrm{W} / \mathrm{m}^{2}\right)\end{array}$ & $\begin{array}{c}\text { Peak flux } \\
\text { approx. }\left(\mathrm{W} / \mathrm{m}^{2}\right)\end{array}$ & $\begin{array}{l}\text { Difference } \\
(\%)\end{array}$ & $\begin{array}{l}\text { Average } \\
\text { flux MC } \\
\left(\mathrm{W} / \mathrm{m}^{2}\right)\end{array}$ & $\begin{array}{c}\text { Average flux } \\
\text { approx. }\left(\mathrm{W} / \mathrm{m}^{2}\right)\end{array}$ & $\begin{array}{l}\text { Difference } \\
(\%)\end{array}$ \\
\hline$(-6.8,27.5)$ & 6936 & 6946 & 0.14 & 410.0 & 410.1 & 0.04 \\
\hline$(15.9,30.2)$ & 5674 & 5581 & 1.65 & 401.7 & 401.8 & 0.04 \\
\hline$(0,34.1)$ & 6288 & 6260 & 0.44 & 400.6 & 401.8 & 0.28 \\
\hline$(0,49.9)$ & 4213 & 4169 & 1.05 & 383.7 & 383.5 & 0.04 \\
\hline$(8.1,56.3)$ & 3513 & 3464 & 1.41 & 377.4 & 377.3 & 0.02 \\
\hline$(14.2,66.7)$ & 2701 & 2616 & 0.77 & 367.9 & 368.6 & 0.19 \\
\hline$(0,88.5)$ & 1765 & 1716 & 2.78 & 354.9 & 354.1 & 0.27 \\
\hline$(-67.5,167.0)$ & 463 & 438 & 5.57 & 248.2 & 242.1 & 2.46 \\
\hline$(-23.5,205.7)$ & 379 & 361 & 4.73 & 223.4 & 218.4 & 2.25 \\
\hline$(-7.8,206.9)$ & 381 & 363 & 4.65 & 224.1 & 219.1 & 2.25 \\
\hline
\end{tabular}




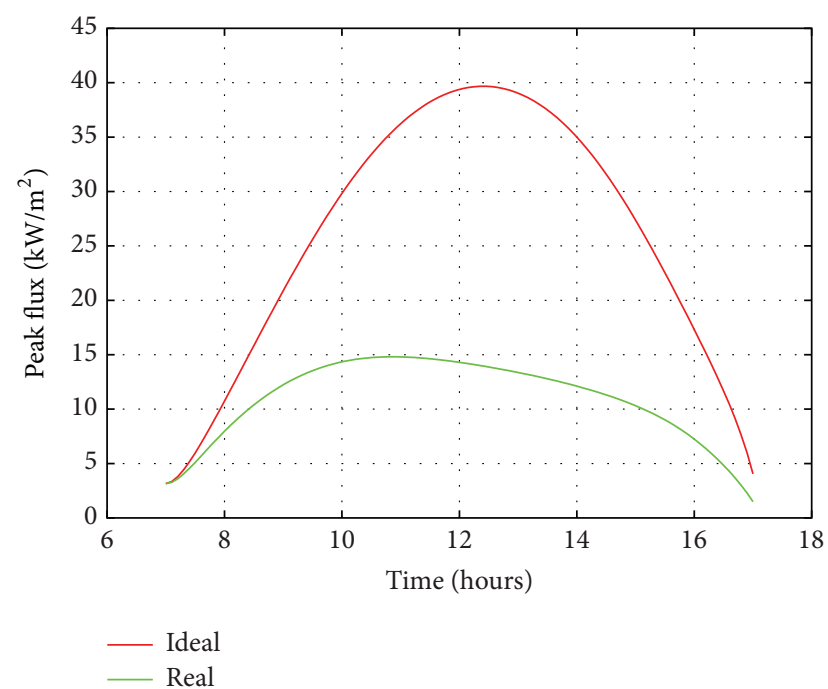

FIgure 9: Peak flux comparison for the 5 heliostats of Figure 8, with no drift (ideal) and with drift (real), for the summer solstice.

individual heliostats, which tend to display much less regular distributions. Thus, this approximation is intended for the simulation of many heliostats simultaneously.

Another point to be mentioned is that the locations of the centroid of the flux distribution and its peak may not coincide in very astigmatic situations. As mentioned previously, in this work drift is calculated from the impact point of the central ray, which also does not necessarily coincide with either of the former points. This may be particularly accentuated for large incidence angles, that is, very inclined heliostats (large $\psi$ ) or nonflat receivers. However, as has been discussed elsewhere [9], for north field configurations with flat receivers, the distance between these points is relatively small in comparison with the displacements caused by drift. For other kinds of configurations the model should be further developed.

From the above discussion the limitations of the code to reproduce actual results from experiments are clear. The aim of this methodology is rather to serve as a parametric tool to simulate different drift scenarios and to evaluate the impact in plant performance. Also, the present modeling tool can be very useful to study the effect of different advanced heliostat control strategies $[5,6,18]$. Sometimes a uniform flux is sought, but in other cases specialized flux distributions may be sought for some applications. Traditional simulation methods are based on the assumption of Gaussian tracking errors, which may in some cases be very inaccurate, especially for small to medium size heliostat fields. In contrast, the present proposal has the capability to address situations where the heliostat dynamics is important.

\section{Conclusions}

In the present work a modeling tool has been presented for calculation of central receiver concentrated flux distributions, which includes drift effects, unlike any previous software. This modeling tool is based on a drift model that includes different geometrical error sources in a rigorous manner [8] and on an analytic approximation [20] for the individual flux distribution of a heliostat.

This model has been applied to a group of heliostats of a real field, and the accuracy of the flux distribution approximation has been checked for a range of distances to the tower, with excellent results, against SolTrace. Some examples of drift calculations have been presented as well as the resulting flux distribution of five heliostats experience drift along the day. It can be observed that the resulting peak fluxes differ strongly from those obtained assuming the ideal case without drift. The time evolution of quantities like peak flux, average flux, or flux uniformity can be easily calculated with this model. It is clear, from the presented examples, that flux distribution may become very irregular under some circumstances, which rules out the possibility of analyzing them by simply considering the addition of a Gaussian tracking error to the optical errors of the heliostat, as is conventionally done [24]. This latter approach can be useful for studying yearly averages, but not when dynamic effects are important, like in the design of advanced control strategies for heliostats.

\section{Competing Interests}

The authors declare that they have no competing interests.

\section{Acknowledgments}

Luis O. Lara-Cerecedo and Isaias Moreno-Cruz acknowledge the support from CONACYT, Mexico, through graduate scholarships. This work was partially supported by Grant 123767, from CONACYT.

\section{References}

[1] O. Behar, A. Khellaf, and K. Mohammedi, "A review of studies on central receiver solar thermal power plants," Renewable and Sustainable Energy Reviews, vol. 23, pp. 12-39, 2013.

[2] M. Romero, R. Buck, and J. E. Pacheco, "An update on solar central receiver systems, projects, and technologies," Journal of Solar Energy Engineering, vol. 124, no. 2, pp. 98-108, 2002.

[3] A. F. Hildebrandt and L. L. Vant-Hull, "Power with heliostats," Science, vol. 197, no. 4309, pp. 1139-1146, 1977.

[4] V. A. Baum, R. R. Aparasi, and B. A. Garf, "High power solar installations," Solar Energy, vol. 1, no. 1, pp. 6-12, 1957.

[5] A. Salomé, F. Chhel, G. Flamant, A. Ferrière, and F. Thiery, "Control of the flux distribution on a solar tower receiver using an optimized aiming point strategy: application to THEMIS solar tower," Solar Energy, vol. 94, pp. 352-366, 2013.

[6] L. Roca, A. de la Calle, and L. J. Yebra, "Heliostat-field gainscheduling control applied to a two-step solar hydrogen production plant," Applied Energy, vol. 103, pp. 298-305, 2013.

[7] K.-K. Chong and M. H. Tan, "Comparison study of two different sun-tracking methods in optical efficiency of heliostat field," International Journal of Photoenergy, vol. 2012, Article ID 908364, 10 pages, 2012.

[8] M. Escobar-Toledo, C. A. Arancibia-Bulnes, C. Iriarte-Cornejo et al., "Heliostat image drift behavior for different error sources," Journal of Renewable and Sustainable Energy, vol. 6, no. 2, Article ID 023117, 2014.

[9] L. A. Díaz-Félix, M. Escobar-Toledo, J. Waissman, N. PitalúaDíaz, and C. A. Arancibia-Bulnes, "Evaluation of heliostat field 
global tracking error distributions by Monte Carlo simulations," Energy Procedia, vol. 49, pp. 1308-1317, 2014.

[10] M. Guo, Z. Wang, J. Zhang, F. Sun, and X. Zhang, "Accurate altitude-azimuth tracking angle formulas for a heliostat with mirror-pivot offset and other fixed geometrical errors," Solar Energy, vol. 85, no. 5, pp. 1091-1100, 2011.

[11] M. Guo, Z. Wang, J. Zhang, F. Sun, and X. Zhang, "Determination of the angular parameters in the general altitude-azimuth tracking angle formulas for a heliostat with a mirror-pivot offset based on experimental tracking data," Solar Energy, vol. 86, no. 3, pp. 941-950, 2012.

[12] A. Kribus, I. Vishnevetsky, A. Yogev, and T. Rubinov, "Closed loop control of heliostats," Energy, vol. 29, no. 5-6, pp. 905-913, 2004.

[13] M. Berenguel, F. R. Rubio, A. Valverde et al., "An artificial visionbased control system for automatic heliostat positioning offset correction in a central receiver solar power plant," Solar Energy, vol. 76, no. 5, pp. 563-575, 2004.

[14] K. W. Stone and S. A. Jones, "Analysis of solar two heliostat tracking error sources," Tech. Rep. SAND99-0239C, Sandia National Laboratories, Albuquerque, NM, USA, 1999.

[15] S. A. Jones and K. W. Stone, "Analysis of strategies to improve heliostat tracking at solar two," Tech. Rep. SAND99-0092C, Sandia National Laboratories, Albuquerque, NM, USA, 1999.

[16] N.-S. Pai, H.-T. Yau, T.-H. Hung, and C.-P. Hung, "Application of CMAC neural network to solar energy heliostat field fault diagnosis," International Journal of Photoenergy, vol. 2013, Article ID 938162, 8 pages, 2013.

[17] M. Escobar-Toledo, C. A. Arancibia-Bulnes, M. I. Peña-Cruz et al., "Analysis of drift phenomena in heliostat images," in Proceedings of the 6th International Conference on Energy Sustainability (ASME '12), paper ES2012-91187, pp. 385-392, San Diego, Calif, USA, July 2012.

[18] C. Iriarte-Cornejo, C. A. Arancibia-Bulnes, I. Salgado-Tránsito, J. Waissman, R. E. Cabanillas, and C. A. Estrada, "Compensation of heliostat drift by seasonal sampling," Solar Energy, vol. 105, pp. 330-340, 2014.

[19] P. Garcia, A. Ferriere, and J.-J. Bezian, "Codes for solar flux calculation dedicated to central receiver system applications: a comparative review," Solar Energy, vol. 82, no. 3, pp. 189-197, 2008.

[20] F. J. Collado, A. Gómez, and J. A. Turégano, "An analytic function for the flux density due to sunlight reflected from a heliostat," Solar Energy, vol. 37, no. 3, pp. 215-234, 1986.

[21] F. J. Collado, "Quick evaluation of the annual heliostat field efficiency," Solar Energy, vol. 82, no. 4, pp. 379-384, 2008.

[22] M. D. Walzel, F. W. Lipps, and L. L. Vant-Hull, "A solar flux density calculation for a solar tower concentrator using a twodimensional hermite function expansion," Solar Energy, vol. 19, no. 3, pp. 239-253, 1977.

[23] J. A. Duffie and W. A. Beckman, Solar Engineering of Thermal Processes, John Wiley \& Sons, Hoboken, NJ, USA, 4th edition, 2013.

[24] A. Rabl, Active Solar Collectors and Their Applications, Oxford University Press, New York, NY, USA, 1985. 

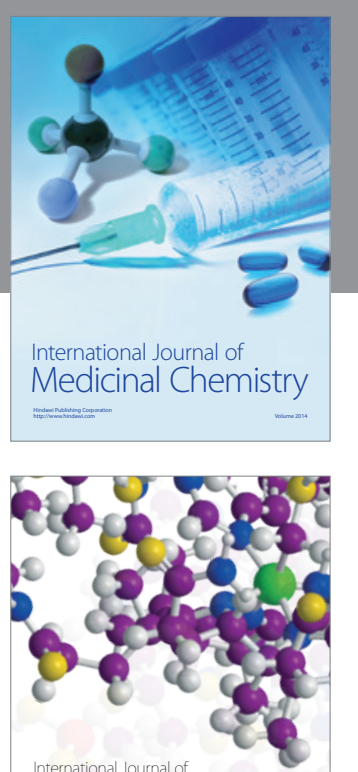

Carbohydrate Chemistry

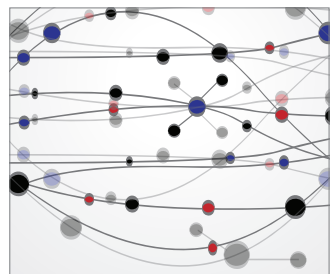

The Scientific World Journal
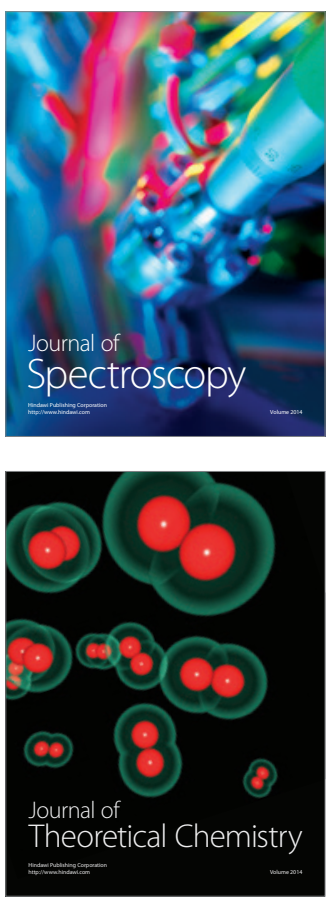
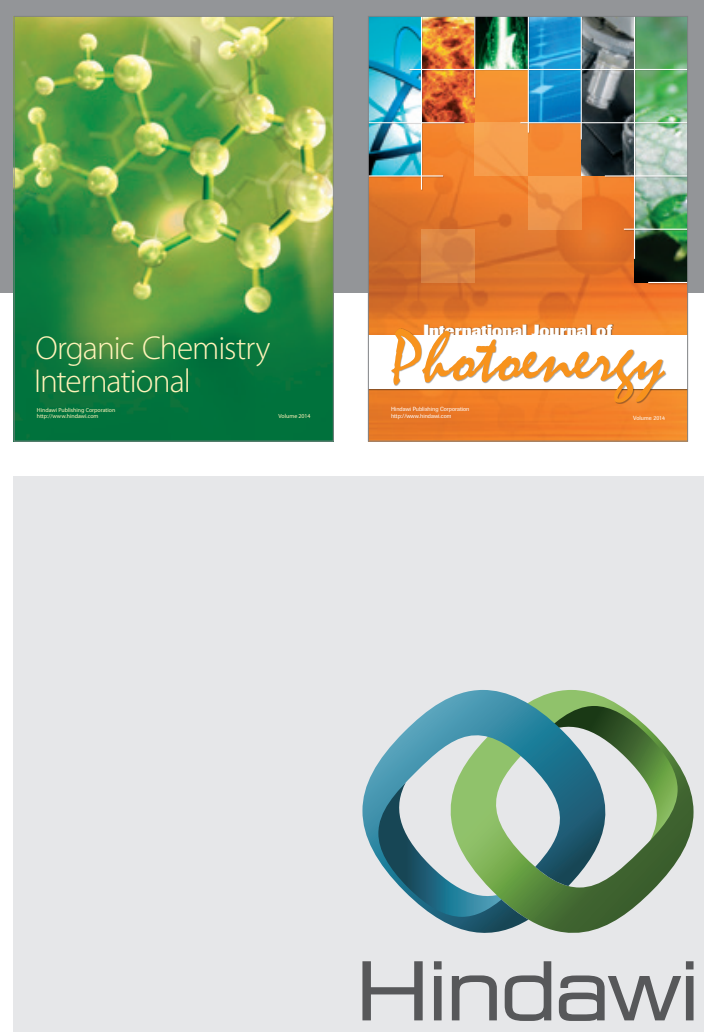

Submit your manuscripts at

http://www.hindawi.com

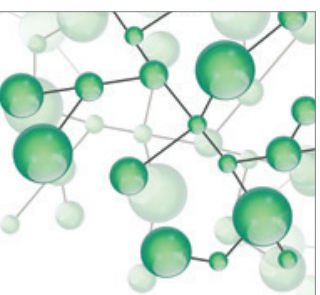

International Journal of

Inorganic Chemistry

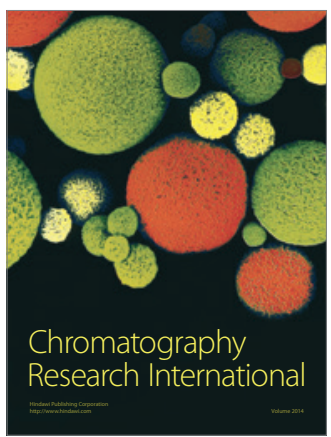

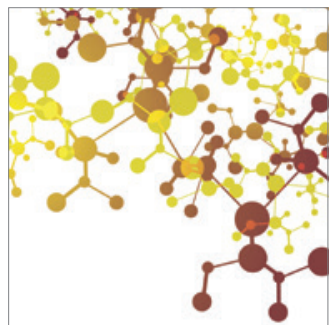

Applied Chemistry
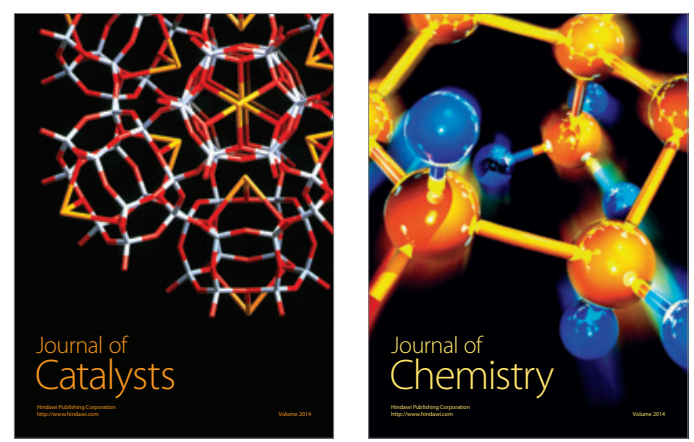
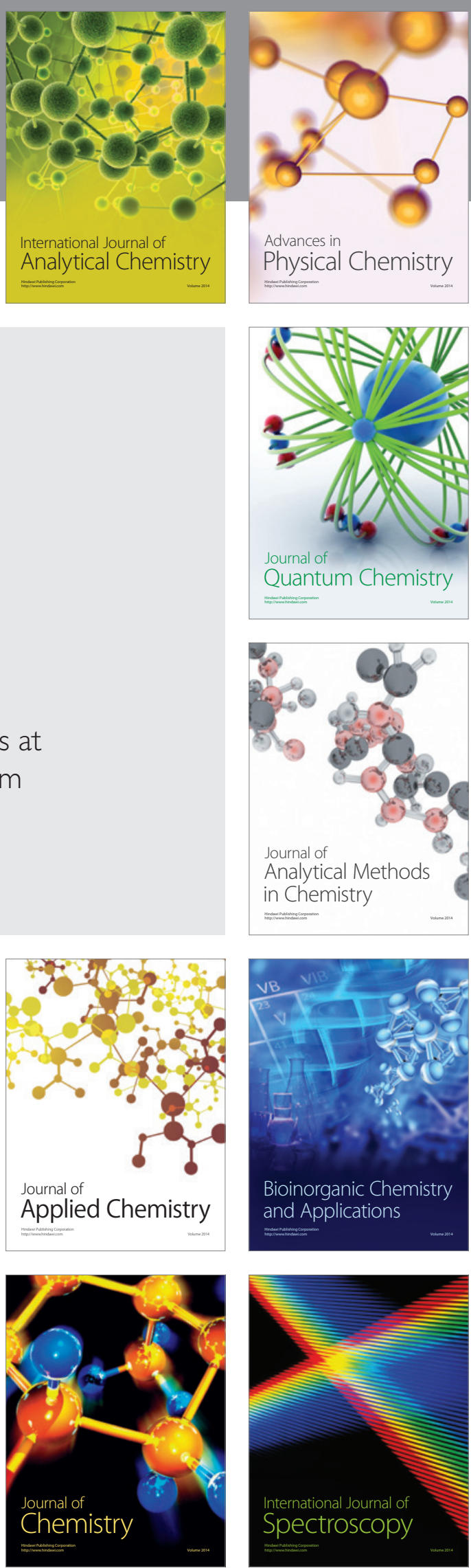